Article

\title{
Terahertz Displacement Sensing Based on Interface States of Hetero-Structures
}

\author{
Lan-Lan Xu ${ }^{1,2}$, Ya-Xian Fan $1,3, * \mathbb{1}$, Huan Liu ${ }^{1}$, Tao Zhang ${ }^{1}$ and Zhi-Yong Tao $1,3, * \mathbb{1}$ \\ 1 Guangxi Key Laboratory of Wireless Wideband Communication and Signal Processing, \\ Guilin University of Electronic Technology, Guilin 541004, China; lanlanxu@hrbeu.edu.cn (L.-L.X.); \\ huanliu@guet.edu.cn (H.L.); zt@guet.edu.cn (T.Z.) \\ 2 Key Lab of In-Fiber Integrated Optics, Ministry Education of China, Harbin Engineering University, \\ Harbin 150001, China \\ 3 Academy of Marine Information Technology, Guilin University of Electronic Technology, \\ Beihai 536000, China \\ * Correspondence: yxfan@guet.edu.cn (Y.-X.F.); zytao@guet.edu.cn (Z.-Y.T.)
}

Received: 10 July 2020; Accepted: 27 July 2020; Published: 28 July 2020

check for updates

\begin{abstract}
Herein, we propose a nano displacement sensor based on the interface state of a terahertz hetero-structure waveguide. The waveguide consists of two periodically corrugated metallic tubes with different duty ratios, which can result in similar forbidden bands in their frequency spectra. It was found that the topological properties of these forbidden bands are different, and the hetero-structure can be formed by connecting these two waveguides. In the hetero-structure waveguide, the interface state of an extraordinary transmission can always arise within the former forbidden bands, the peak frequency of which is highly dependent on the cavity length at the interface of the two periodic waveguides. So, by carefully designing the structure's topological property, the hetero-structure waveguide can be efficiently used to produce a displacement sensor in the $\mathrm{THz}$ frequency range. The simulations show that the resolution of the displacement can be as small as $90 \mathrm{~nm}$ and the sensitivity can reach over $1.2 \mathrm{GHz} / \mu \mathrm{m}$. Such a sensitive interface state of the proposed hetero-structure waveguide will greatly benefit $\mathrm{THz}$ applications of functional devices, including not only displacement sensors but also switches with high extinction ratios, tunable narrow-band filters, and frequency division multiplexers.
\end{abstract}

Keywords: resonances; periodic waveguides; reflection phases; topological properties

\section{Introduction}

In recent years, with the development of ultra-fast technologies, the research on terahertz $(\mathrm{THz})$ technology has seen unprecedented progress [1-4]. THz waves have many unique advantages over the electromagnetic waves in the other bands, which allow $\mathrm{THz}$ technology to have considerable applications in some important areas, such as military, astronomy, radar, and medicine [5-8]. Due to its strong penetrability and low photonic energy, $\mathrm{THz}$ imaging technology will replace $\mathrm{X}$-rays in medical examinations and will not cause harm to the human body [9]. It can also be used for detection in complex environments and identification of plastic weapons [10]. The characteristic spectra of many biomolecules and chemicals are also located in the THz band, including many drugs. So, it is also useful to employ $\mathrm{THz}$ waves in substance identification and biomedical research [11]. The broadband characteristics of $\mathrm{THz}$ waves result in their predictable applications in communication fields, and their wireless transmission speed can reach tens of $\mathrm{Gb} / \mathrm{s}[12,13]$. In the upcoming $6 \mathrm{G}$ Internet of Things, $\mathrm{THz}$ waves are also considered to be good candidates in short-range, bandwidth-aggressive services such as in the smart home scenario [14]. In THz systems, functional devices are essential to applications, 
including, of cause, displacement sensors. For example, in THz imaging, displacement sensing can correct the errors of relative motion between objects and probes, and it is expected to be adopted in calibrating the amplitude of characteristic peaks for different substances. In this era of interconnection of all things, most communications depend on $\mathrm{THz}$ waves, and displacement sensors can be integrated into smart devices to monitor and control their position changes.

The micro-nano displacement sensor as a device for high-precision monitoring has been used in automotive industry [15], small-scale manipulation [16,17], construction [18,19], micro-grippers [20], physiological sensing [21,22], and so on. The micro-nano displacement sensing technology of the communication optical band has been developed over many years, including plasmonic slot metamaterials [23], Fabry-Pérot interferometers [24], photonic crystal fibers [25], and so on. In 2011, Liu et al. reported a sensing structure with double-fiber Bragg gratings [26]. In 2014, Qu et al. presented an interferometric fiber-optic bending/micro-displacement sensor based on a plastic dual-core fiber with one end coated by a silver mirror [27]. In 2016, Gao et al. realized an optical displacement sensor based on anti-resonant reflecting guidance in a capillary covered hollow-core fiber [28]. However, a $\mathrm{THz}$ micro-nano displacement sensor of waveguide types that can be applied in $\mathrm{THz}$ systems without additional optical devices has not been reported yet. It will be very intriguing to realize $\mathrm{THz}$ micro-nano displacement sensing with high precision, small size, and easy integration. Hypersensitive $\mathrm{THz}$ displacement sensors could expand the applications of $\mathrm{THz}$ technology and pave the way for $\mathrm{THz}$ micro-nano positioning in the future smart life of all things connected.

Here, we propose a THz displacement sensor based on the hetero-structure waveguide, which can provide a sensitive spectrum response to a tiny displacement. Hetero-structures consist of semiconductor materials with different forbidden bands [29-31], which have been developed for artificial structures for years [32-36]. Further, the waveguides in the $\mathrm{THz}$ frequency range have been investigated numerically for functional devices $[37,38]$. The proposed hetero-structure contains a cylindrical waveguide with two different periodic corrugations on the wall. Based on their different topological properties, these two periodicities are used to generate an interface state, which can be recognized by a very narrow transmitted peak in its spectrum. It was found that the transmitted peak highly depends on the cavity length at the interface of two periodicities. Increasing the cavity length can result in red shift of the peak, which is very promising for use in fabricating a sensitive $\mathrm{THz}$ sensor. In the following section, the hetero-structure waveguide and its arising interface state are demonstrated. There is a transmitted peak arising in the former forbidden band due to the interface of the two periodic structures. The hetero-structure waveguide composed of two opaque periodic tubes become transparent at a certain frequency. The different topological properties of the two periodic structures can be explained by their reflection phases at the interface. The displacement THz sensor is proposed in Section 3 and the sensing performance is discussed in detail. Finally, the major findings about the super-high resolution and sensitivity are summarized.

\section{Hetero-Structure Waveguides}

Hetero-structure waveguides are usually combinations of multiple different tubes with unequal topological band gaps. Between each waveguide of the hetero-structures, interface states can always arise to produce an extraordinary transmitted peak in the former forbidden bands of periodic waveguides, which is very promising for high-resolution sensing due to its very narrow line width. Here, we propose a very simple corrugated waveguide system to demonstrate a $\mathrm{THz}$ micro-nano displacement sensor with very high precision. In waveguides with periodic wall corrugations, frequency gaps will appear around the resonant frequencies, in which the electromagnetic waves cannot pass through the structures. Bragg resonance will happen when the longitudinal wavenumbers of the same transverse modes satisfy the matching condition [39]. Connecting two periodic waveguides with similar Bragg gaps can result in an interface state with a very sharp transmitted peak in the overlapping frequency band gap when their topologies are different. These two waveguides have the same average radius $r_{0}$ and period $\Lambda$, the transmission spectra and geometry structures of which are 
shown in Figure 1a-c. The duty ratio refers to the proportion of wide radius part in a period length, and it has been proved that different duty ratios can introduce different topological band gaps [40]. We selected different duty ratios 0.8 and 0.4 for Waveguides A and B, respectively, to achieve the different topologies.

In a hollow metallic cylindrical periodic waveguide, the lowest-order Bragg resonance happens at

$$
f_{r}=\frac{c}{2 \pi} \sqrt{\frac{k_{r}^{2}}{r_{0}^{2}}+\left(\frac{\pi}{\Lambda}\right)^{2}}
$$

for transverse magnetic (TM) waves with $k_{r}=2.4048$. To set the Bragg resonance around $1 \mathrm{THz}$, we selected the geometry parameters of the periodic waveguides as $R_{\mathrm{I}}=220 \mu \mathrm{m}, R_{\mathrm{II}}=180 \mu \mathrm{m}$, and $\Lambda=183 \mu \mathrm{m}$ with $r_{0}=\left(R_{\mathrm{I}}+R_{\mathrm{II}}\right) / 2=200 \mu \mathrm{m}$, where $R_{\mathrm{I}}$ and $R_{\mathrm{II}}$ are the wide and narrow radii of the structures, respectively. The hetero-structure waveguide was obtained by directly connecting the wide tube of Waveguide A and the narrow tube of Waveguide B.

We employed finite element method simulations on the waveguide structures with an axisymmetric model in COMSOL Multiphysics software. In the simulations, the refractive index of inside air was set to be 1 , and perfect electrical boundary conditions were assigned to the walls of these three waveguides. As the THz source, a first-order TM mode was excited at the inlet of each waveguide, and the radiation condition was assigned to the outlet boundary. The $0.8-1.1 \mathrm{THz}$ frequency range was selected with a step of $0.001 \mathrm{THz}$, and the electromagnetic fields were calculated in the whole waveguide.
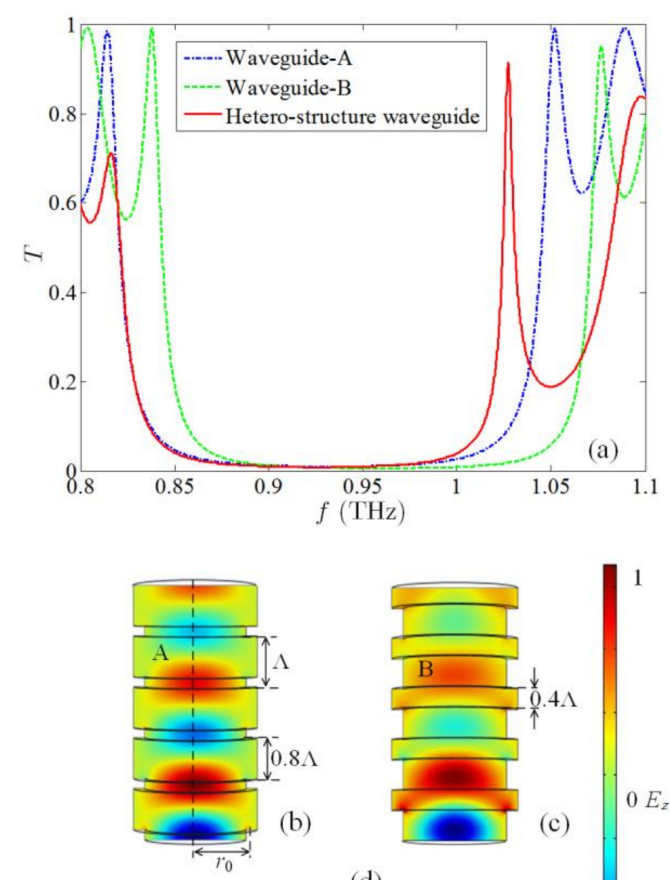

(d)

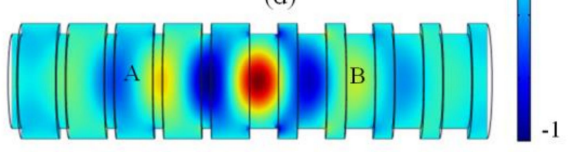

Figure 1. Transmission spectrum and electric field $E_{z}$ components of Waveguides $\mathrm{A}, \mathrm{B}$, and the hetero-structure of their combination. (a) The blue dash-dot, green dash, and red solid lines are the transmission spectra of Waveguides $A$ and $B$ and the hetero-structure, respectively. The electric fields $E_{z}$ of Waveguides A (b) and B (c) and the hetero-structure (d) at $1.027 \mathrm{THz}$ illustrate the energy attenuation and localization at the interface of the structure. The electric fields were normalized by their own maximum in each waveguide. 
Defining the transmission coefficient $T$ as the ratio of optical powers of the outlet and inlet, we calculated the transmission spectra of Waveguides A and B and the hetero-structure waveguide, and depicted them by the dash-dot, dashed, and solid lines in Figure 1a. It shows that Waveguide A has a band gap around $0.838-1.038 \mathrm{THz}$, and Waveguide B's is around 0.848-1.051 THz. The smaller duty ratio of Waveguide $B$ leads to a small frequency shift of the whole Bragg gap to a higher frequency. However, these two waveguides have a common forbidden band in the frequency range of $0.848-1.038 \mathrm{THz}$, in which the $\mathrm{THz}$ radiation cannot pass through either Waveguide $\mathrm{A}$ or $\mathrm{B}$. The frequency of $1.027 \mathrm{THz}$ falls in this common forbidden band-that is to say, a $\mathrm{THz}$ wave at this frequency cannot pass through either of these two waveguides-but what a surprise that we find an extraordinary transmitted peak with a center frequency of $1.027 \mathrm{THz}$ in the spectrum of the hetero-structure waveguide. The forbidden band becomes much wider than that of either of the two waveguides.

To verify the interface state arising, we also simulated the electric field distributions for these three different waveguides, and the $E_{z}$ components of the electric fields at $1.027 \mathrm{THz}$ are depicted in Figure $1 b-d$ for Waveguides A and B and the hetero-structure, respectively. The electric fields were normalized by their own maxima in the figure. The $\mathrm{THz}$ waves enter the waveguide from the bottoms of Waveguides A and B in Figure $1 \mathrm{~b}, \mathrm{c}$ respectively. It can be observed that the $\mathrm{THz}$ wave decays along the direction of propagation. Fortunately, the frequency is very close to the edge of the forbidden bands. So, we find that Waveguide $B$ with a smaller duty ratio is much more effective for $\mathrm{THz}$ attenuation. In any case, the THz radiation at $1.027 \mathrm{THz}$ cannot pass through either Waveguide A or B. When we connect these two waveguides and excite a THz wave with the first TM mode at the left side of the hetero-structure waveguide, as shown in Figure 1d, the situation turns out to be totally different. The $\mathrm{THz}$ waves accumulate around the interface of Waveguides A and B, which is known as the interface state. Due to the energy accumulation, the former opaque waveguides become transparent to a special frequency $\mathrm{THz}$ wave, as shown in Figure 1a. It is because of the normalization to the maximum electric field that the $E_{z}$ component at the outlet is smaller than 1 . Based on the transmission spectrum and the electric field distribution, we confirm the interface state arising in the proposed hetero-structure waveguide.

The remarkable transmission feature is due to the different topological properties of the two waveguides, which can be identified by the Zak phases of the two Bragg bands [40]. It is also more convenient to investigate the reflection phase of each waveguide at the interface. If the phases have the same sign, the related Bragg gaps are of a similar topology. Otherwise, they are topologically different, and the interface state arises.

To achieve the reflection phase of each waveguide, we connected a straight tube to the corrugated one and simulated the $E_{z}$ component of electric fields with an incident TM mode from the straight tube at $1.027 \mathrm{THz}$. A straight tube with a length of $1000 \mu \mathrm{m}$ and radius of $200 \mu \mathrm{m}$ was connected to the wide radius port of Waveguide A. For Waveguide B, it was connected to the narrow radius port. Thus, the reflection properties at the interface of each waveguide could be observed. The same straight tube with a perfect electrical boundary at the other end was also simulated for reference. The reflected components of the electric fields are shown in Figure $2 \mathrm{a}-\mathrm{c}$ for Waveguide A, the perfect electrical boundary, and Waveguide B, respectively. For convenience, we also depict the amplitude of $E_{z}$ by solid lines. With the aid of the added dashed line, we can conclude that the reflection phase of Waveguide $\mathrm{A}$ is delayed while that of Waveguide B is advanced when considering the perfect electrical boundary reference as a zero-phase case. The reflection phases of Waveguides A and B have opposite signs, indicating the topological difference of the two Bragg gaps. So, the interface state arises at $1.027 \mathrm{THz}$, where we found a narrow transmitted peak in the spectrum. Such an extraordinary peak with very narrow line width would be a good candidate for $\mathrm{THz}$ sensing applications. 


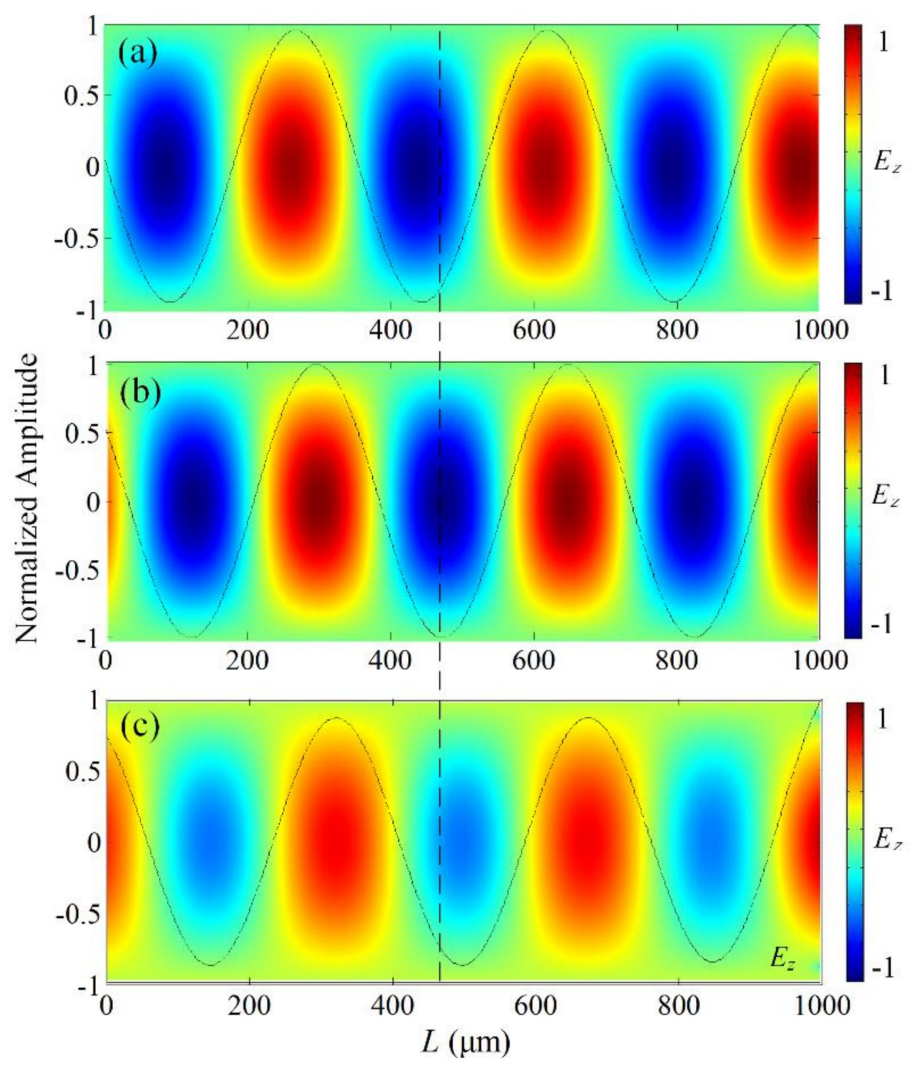

Figure 2. $E_{z}$ components of reflected electric fields at $1.027 \mathrm{THz}$ for (a) Waveguide $\mathrm{A}$, (b) the perfect electrical boundary, and (c) Waveguide B. The reflected phase of Waveguide A is delayed while that of Waveguide $\mathrm{B}$ is advanced relative to the $\mathrm{THz}$ waves reflected by the perfect electrical boundary.

\section{Micro-Nano Displacement Sensing}

To realize micro-nano displacement sensing, we propose the hetero-structure waveguide (Figure 3a) composed of two tubes with average inner radius $200 \mu \mathrm{m}$ and period $183 \mu \mathrm{m}$ based on the above analysis. The tube wall is suggested to be $10 \mu \mathrm{m}$ thick silver (Ag), which has low loss in the THz frequency range. So, the outer radii are $190 \mu \mathrm{m}$ and $230 \mu \mathrm{m}$ for the narrow and wide parts, respectively. According to the duty ratios, the lengths of the wide and narrow tubes are $146.4 \mu \mathrm{m}$ and $36.6 \mu \mathrm{m}$, respectively, for Waveguide A, whereas they are $73.2 \mu \mathrm{m}$ and $109.8 \mu \mathrm{m}$ for Waveguide B. To fabricate an integrated device, we have to increase the length of the wide tube at the right end of Waveguide A and add a ring at the left end of Waveguide B. The increased length is $276 \mu \mathrm{m}$ and the outer radius of the ring should be $220 \mu \mathrm{m}$ to make sure that they can be connected. The length can be mechanically changed within the range of 20-120 $\mu \mathrm{m}$. Although the measurement cannot start from $0 \mu \mathrm{m}$ due to the length of the narrow tube in Waveguide $B$, the measurement range of displacement can still reach $100 \mu \mathrm{m}$. The number of periods in each waveguide is 5 .

We also performed simulations on different combinations of duty ratios. The duty ratios of Waveguides A and B were selected from 0.1 to 0.9 in intervals of 0.1 . The results show that all connections of different duty ratios can create a similar extraordinary transmitted peak, that is to say, the interface state between the two periodic waveguides always arises in the former Bragg gaps due to the different topologies. However, the bandwidth and frequency shift of the peaks highly rely on the duty ratios. Only the duty ratio combination of 0.8 and 0.4 can result in the narrowest bandwidth and maximum displacement, which could be of extreme benefit for practical applications. So, the proposed structure with duty ratios 0.8 and 0.4 was analyzed in detail for $\mathrm{THz}$ displacement sensing.

In the displacement sensing, we first fix Waveguide $A$, then adjust the Waveguide $B$ to a relative position to be measured, and finally fix Waveguide B to that position. In this way, Waveguide B 
moves relative to Waveguide A, which increases the length of the cavity between the two waveguides. The $\mathrm{THz}$ wave is incident from the narrow radius of Waveguide $\mathrm{A}$ and emitted from the wide radius of Waveguide B. The frequency of the transmitted peak shifts with the displacement, so that the hetero-structure waveguide realizes displacement sensing. The simulated transmission for different displacements is depicted in Figure $3 b$ by different lines. The center frequency of the transmitted peak is $1.002 \mathrm{THz}$ when the displacement is $20 \mu \mathrm{m}$ in the hetero-structure waveguide. When Waveguide $B$ moves away from Waveguide $\mathrm{A}$, the length of the middle cylindrical waveguide increases and the peak frequency shifts to the low frequency range. All the transmissions are above 0.85 , and the highest transmission of 0.948 is obtained when the displacement is $120 \mu \mathrm{m}$.

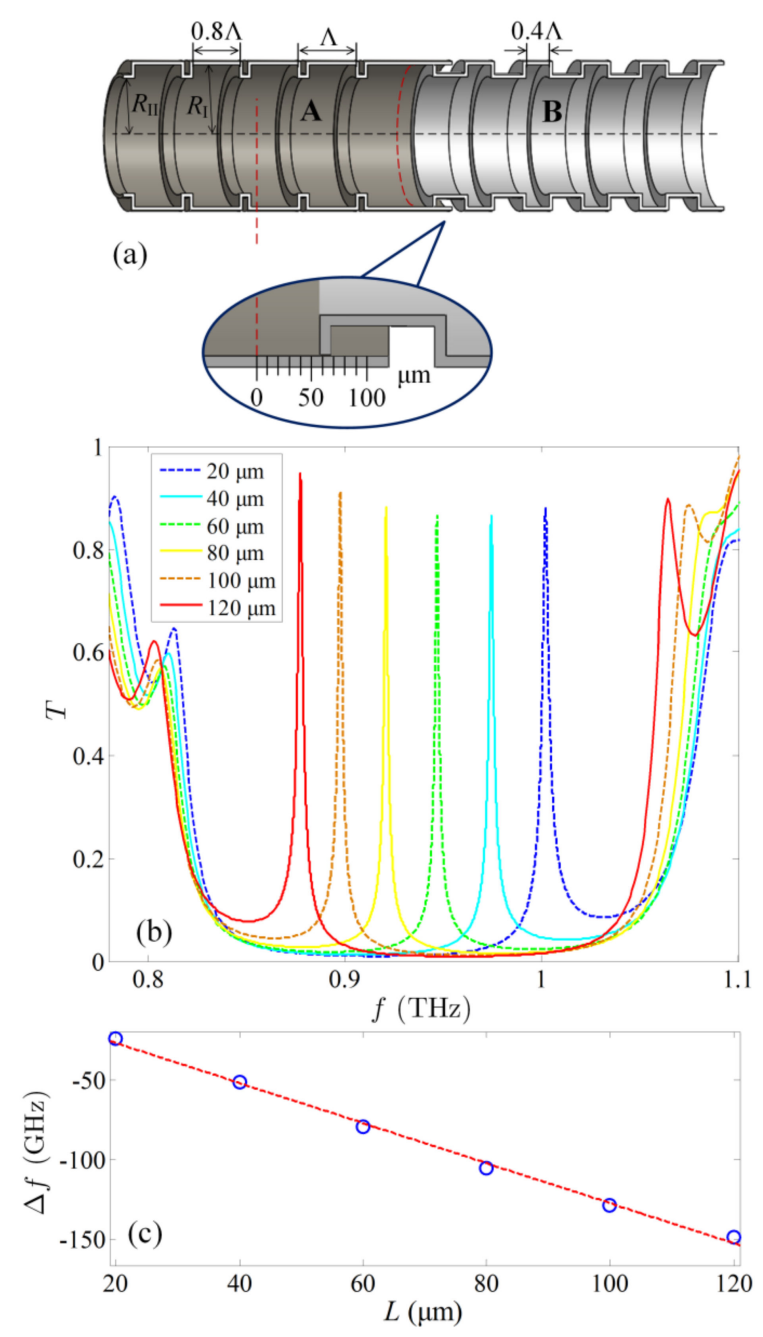

Figure 3. The THz micro-nano displacement sensor and its sensing performance. (a) Structure diagram of the hetero-structure waveguide sensor. The dark and light gray parts are Waveguides A and B, respectively. The red dashed line denotes the zero-displacement point and the inset is a magnification of the shifting structure with scales. (b) Transmitted peaks for the different displacements according to the scales. (c) Peak frequency shifting with increasing displacement.

To measure the displacement by $\mathrm{THz}$ waves, we define the frequency shift $\Delta f$ from the frequency of $1.026 \mathrm{THz}$ at $0 \mu \mathrm{m}$. The former peak moves from $1.027 \mathrm{THz}$ to $1.026 \mathrm{THz}$ for the $0 \mu \mathrm{m}$ waveguide because the real dielectric constant of Ag is considered in the simulations. The frequency shift $\Delta f$ according to the displacement is marked in Figure $3 \mathrm{c}$ by the circles, and we performed a linear fit (the dashed line) as follows:

$$
\Delta f=-1.260 \times L
$$


where $L$ is the displacement in micrometers, and the frequency shift is in gigahertz. The fitting results show that when the hetero-structure waveguide is stretched $1 \mu \mathrm{m}$, the transmitted peak moves $1.260 \mathrm{GHz}$ to a lower frequency. When $L=80 \mu \mathrm{m}$, the narrowest bandwidth is $2.580 \mathrm{GHz}$, and when $L=20 \mu \mathrm{m}$, the maximum bandwidth is $4.610 \mathrm{GHz}$. The maximum displacement that can be measured by the hetero-structure waveguide is $100 \mu \mathrm{m}$, the adjustable range of frequency is $0.877-1.002 \mathrm{THz}$, and the whole bandwidth is $124.5 \mathrm{GHz}$. So, the sensitivity of such a waveguide-type sensor can reach over $1.2 \mathrm{GHz} / \mu \mathrm{m}$, and the minimum resolvable length is $2.073 \mu \mathrm{m}$ when the displacement $L$ is around $80 \mu \mathrm{m}$.

To realize the proposed structure, there are two ideas for fabrication. The first idea is to make a hollow metallic waveguide with substrates outside, as shown in Figure 4a. The deep lithography process can be employed to machine two half-cylinder polymers, and the metallic materials, such as $\mathrm{Ag}$, can be sputtered on the two parts. To form the tube, we can hold the two parts together and eliminate a thin layer at the end of Waveguide B. The second idea is to produce a corrugated polymer core by 3D printing, as shown in Figure 4b. The two structures both end at the narrow radius parts. A silver film can be coated on the surface of the corrugated core, and a capillary coated with a silver film inside can be used to connect the two waveguides. Thus, the shifting parts are still hollow. When the proposed structure is ready, Waveguides A and B can be fixed to the two holders using epoxy glue [28]. With the aid of a 3D nano-positioning stage or optical micromanipulation, we can assemble the sensor to the test structures or THz systems.

(a)
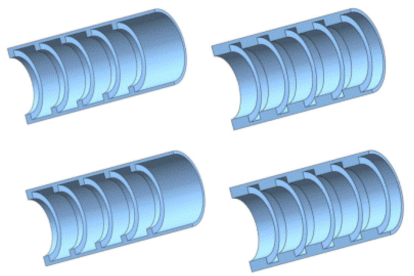

half-cylinder polymers

(b)

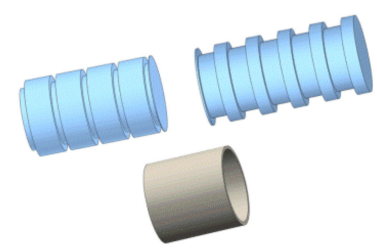

3D-printed polymer cores and a capillary
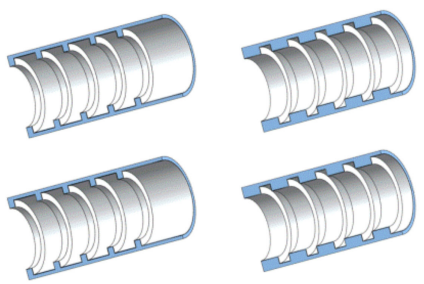

coated by Ag
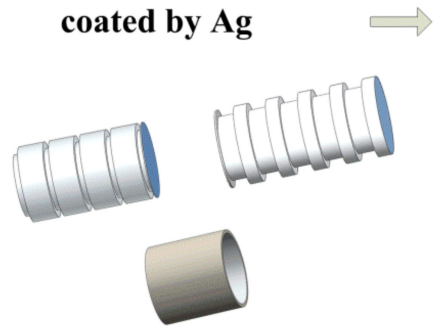

coated by Ag combination
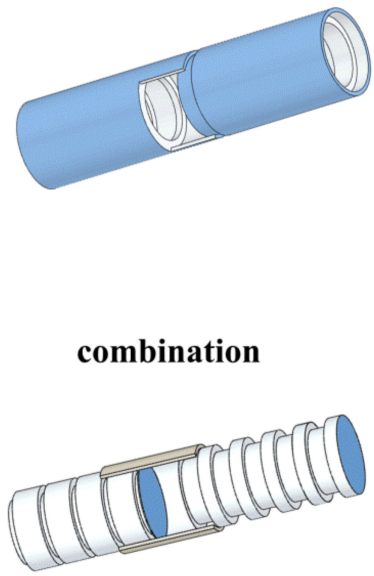

combination

Figure 4. Sketch maps of the device fabrication process with the substrates outside (a) or inside (b).

The number of periods is also an important parameter for the hetero-structure waveguide sensor and can directly affect its resolution. Here we studied the effects of the number of periods on the sensing performance. The number of periods of the two waveguides takes the same value and is denoted by $N$. The value of $N$ ranges from 4 to 9 , while the length $L$ is fixed at $80 \mu \mathrm{m}$. The transmission spectra and the measurement resolutions of the micro-nano displacement sensor are shown in Figure $5 \mathrm{a}, \mathrm{b}$, respectively, in the cases of different $N$. The bandwidth becomes extremely narrow as the number of periods increases, but when the number of periods is greater than 8 , the transmission gets a little bit smaller. When $N=9$, the transmission is just over 0.2. When the number of periods is smaller than 7 , the transmissions are all greater than 0.5 . The variation of the measurement resolution according to 
the number of periods $N$ for the hetero-structure sensor is also depicted in Figure $5 \mathrm{~b}$ by the crosses, with its fitting curve (the solid line) as

$$
R s=7063 \times N^{-5.083}
$$
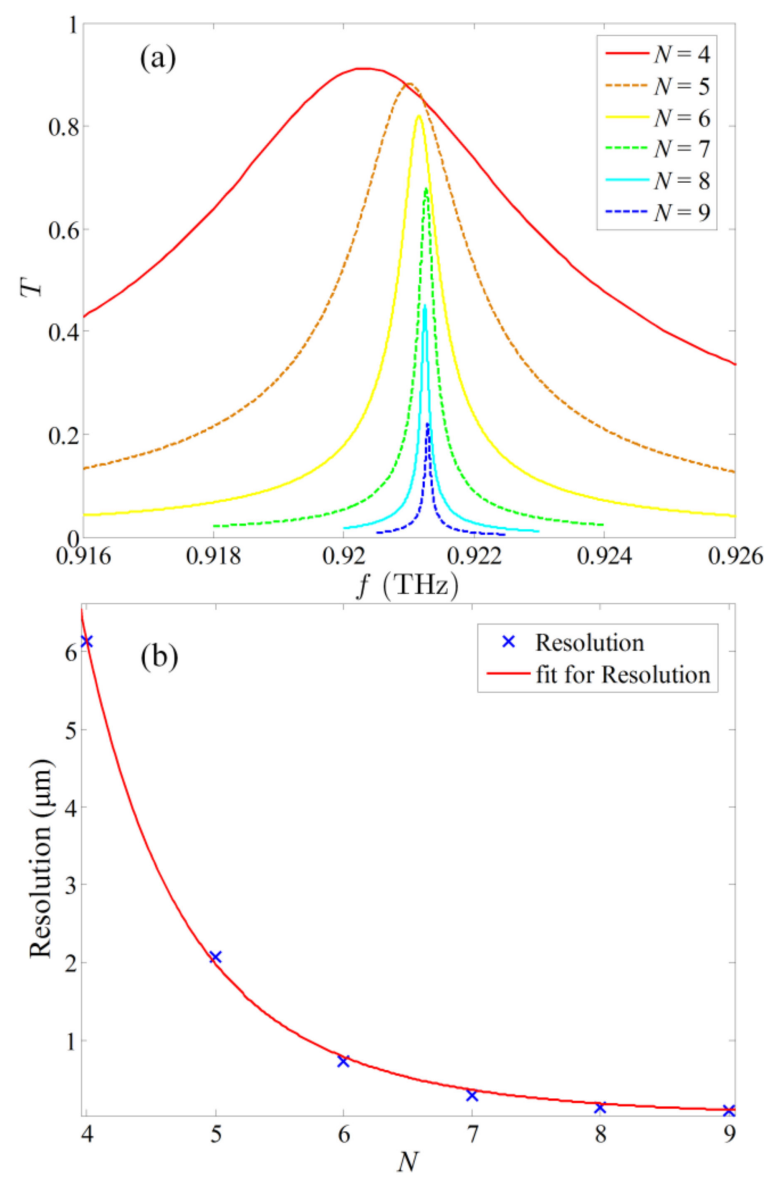

Figure 5. Transmitted peaks (a) and measurement resolutions (b) for different numbers of periods.

The resolution of the sensor is defined as its smallest measurable displacement, which is inversely proportional to the fifth power of $N$. When increasing the number of periods, we can greatly improve the performance of the sensor. The resolution reaches $282 \mathrm{~nm}$ for $N=7$, while it gets as small as $90 \mathrm{~nm}$ for $N=9$. The related sensitivity is over $1.2 \mathrm{GHz} / \mu \mathrm{m}$. This is a highly sensitive $\mathrm{THz}$ nano displacement sensor that can be used in various applications involving accurate displacement measurements. It can also be extended to a wider range of applications when cascade hetero-structure waveguides are considered.

Through simulation of the hetero-structure waveguide, it was proved that the structure can produce a very narrow $\mathrm{THz}$ pulse. By changing the connection length between Waveguides $\mathrm{A}$ and $\mathrm{B}$, the frequency of the transmitted peak moves towards the low frequency range, thus determining the micro-nano displacement sensing function of the proposed structure in the $\mathrm{THz}$ region. For measuring car body paints, a THz transceiver was mounted on a robot [41]. The proposed sensor can be integrated to monitor tiny movements of the mechanical arms without any additional sources. Based on the recorded $\mathrm{THz}$ data, the extracted layer thickness can be corrected. In the Internet of Things, THz functional devices play a key role in communication and intelligence applications [42]. The integrated displacement sensor can not only provide more accurate locations of communication 
nodes, but also monitor subtle changes of smart terminals. The structure design and performance simulations confirmed $\mathrm{THz}$ displacement sensing based on the hetero-structure waveguide.

\section{Conclusions}

We proposed a micro-nano displacement sensor based on hetero-structure waveguides in the $\mathrm{THz}$ frequency range. It is composed of two periodically corrugated waveguides that have similar Bragg forbidden bands. The $\mathrm{THz}$ waves cannot pass through either of these two periodic waveguides in the common frequency range of $0.848-1.038 \mathrm{THz}$. The topological analysis indicates that the Bragg gaps can present different topological properties, which can create an interface state with a very sharp transmitted peak in the $\mathrm{THz}$ spectrum when these two periodic waveguides are connected. Due to the topological difference of the Bragg gaps, the hetero-structure waveguide turns transparent at a certain frequency, where both the waveguides are otherwise opaque. Based on the interface state's induced transmission, we proposed a $\mathrm{THz}$ micro-nano displacement sensor by carefully connecting two corrugated waveguides. When the two waveguides are held by different structures, their relative displacement can be achieved by measuring the shift of transmission peaks, and the resolution can be improved by increasing the number of periods. The proposed hetero-structure waveguide-type sensor has excellent performance, such as a wide measurable range of $100 \mu \mathrm{m}$, minimum resolution of $90 \mathrm{~nm}$, and maximum sensitivity of over $1.2 \mathrm{GHz} / \mu \mathrm{m}$, which allow it various applications in many fields, such as chemical and biomedical sensing, micro-manipulation, imaging, and intelligent control. Besides displacement sensing applications, the proposed interface state of the hetero-structure waveguide can also be applied to other functional devices, such as switches, filters, and frequency division multiplexers.

Author Contributions: Conceptualization, Y.-X.F. and Z.-Y.T.; methodology, H.L. and L.-L.X.; validation, L.-L.X., and H.L.; formal analysis, Y.-X.F., T.Z., and Z.-Y.T.; writing-original draft preparation, L.-L.X.; writing-review and editing, Y.-X.F. and Z.-Y.T.; visualization, L.-L.X.; supervision, Y.-X.F. and Z.-Y.T. All authors have read and agreed to the published version of the manuscript.

Funding: This research was funded by the Dean Project of Guangxi Key Laboratory of Wireless Broadband Communication and Signal Processing, the National Natural Science Foundation of China (11374071), and the Natural Science Foundation of Heilongjiang Province, China (A2018004).

Conflicts of Interest: The authors declare no conflict of interest.

\section{References}

1. Köhler, R.; Tredicucci, A.; Beltram, F.; Beere, H.E.; Linfield, E.H.; Davies, A.G.; Ritchie, D.A.; Iotti, R.C.; Rossi, F. Terahertz semiconductor-heterostructure laser. Nature 2002, 417, 156-159. [CrossRef] [PubMed]

2. Tonouchi, M. Cutting-edge terahertz technology. Nat. Photon. 2007, 1, 97-105. [CrossRef]

3. Thoma, P.; Scheuring, A.; Hofherr, M.; Wünsch, S.; Ilin, K.; Smale, N.; Judin, V.; Hiller, N.; Müller, A.S.; Semenov, A.; et al. Real-time measurement of picosecond $\mathrm{THz}$ pulses by an ultra-fast $\mathrm{YBa}_{2} \mathrm{Cu}_{3} \mathrm{O}_{7-\mathrm{d}}$ detection system. Appl. Phys. Lett. 2012, 101, 142601. [CrossRef]

4. Preu, S.; Mittendorff, M.; Winnerl, S.; Lu, H.; Gossard, A.C.; Weber, H.B. Ultra-fast transistor-based detectors for precise timing of near infrared and THz signals. Opt. Express 2013, 21, 17941-17950. [CrossRef] [PubMed]

5. Cazzoli, G.; Cludi, L.; Buffa, G.; Puzzarini, C. Precise THz measurements of $\mathrm{HCO}^{+}, \mathrm{N}_{2} \mathrm{H}^{+}$, and $\mathrm{CF}^{+}$for astrophysical observations. Astrophys. J. Suppl. Ser. 2012, 203, 1-9. [CrossRef]

6. Yang, Q.; Qin, Y.; Deng, B.; Wang, H.; You, P. Micro-Doppler ambiguity resolution for wideband terahertz radar using intra-pulse interference. Sensors 2017, 17, 993. [CrossRef]

7. Jiang, Y.; Deng, B.; Wang, H.; Qin, Y.; Liu, K. An effective nonlinear phase compensation method for FMCW terahertz radar. IEEE Photon. Technol. Lett. 2016, 28, 1684-1687. [CrossRef]

8. Jiang, X.; Chen, H.; Li, Z.; Yuan, H.; Cao, L.; Luo, Z.; Zhang, K.; Zhang, Z.; Wen, Z.; Zhu, L.; et al. All-dielectric metalens for terahertz wave imaging. Opt. Express 2018, 26, 14132-14142. [CrossRef]

9. Knobloch, P.; Schildknecht, C.; Kleine-Ostmann, T.; Koch, M.; Hoffmann, S.; Hofmann, M.; Rehberg, E.; Sperling, M.; Donhuijsen, K.; Hein, G.; et al. Medical THz imaging: An investigation of histo-pathological samples. Phys. Med. Biol. 2002, 47, 3875-3884. [CrossRef] 
10. Federici, J.F.; Schulkin, B.; Huang, F.; Gary, D.; Barat, R.; Oliveira, F.; Zimdars, D. THz imaging and sensing for security applications-Explosives, weapons and drugs. Semicond. Sci. Technol. 2005, 20, S266-S280. [CrossRef]

11. Fischer, B.M.; Walther, M.; Jepsen, P.U. Far-infrared vibrational modes of DNA components studied by terahertz time-domain spectroscopy. Phys. Med. Biol. 2002, 47, 3807-3814. [CrossRef] [PubMed]

12. Ferraro, A.; Tanga, A.A.; Zografopoulos, D.C.; Messina, G.C.; Ortolani, M.; Beccherelli, R. Guided mode resonance flat-top bandpass filter for terahertz telecom applications. Opt. Lett. 2019, 44, 4239-4242. [CrossRef] [PubMed]

13. Wang, C.; Lu, B.; Lin, C.; Chen, Q.; Miao, L.; Deng, X.; Zhang, J. 0.34-THz Wireless Link Based on High-Order Area Network Applications. IEEE Trans. Terahz. Sci. Technol. 2014, 4, 75-85. [CrossRef]

14. Mao, B.; Kawamoto, Y.; Kato, N. AI-based Joint Optimization of QoS and Security for 6G Energy Harvesting Internet of Things. IEEE Internet Things J. 2020. [CrossRef]

15. Kim, Y.S.; Choi, Y.H.; Lee, J.M.; Noh, J.S.; Kim, J.J.; Bien, F. Fabrication of a novel contactless switch using eddy current displacement sensor for safer vehicle brake system. IEEE Trans. Veh. Technol. 2011, 60, 1485-1495.

16. Li, Y.; Xu, Q. Adaptive sliding mode control with perturbation estimation and PID sliding surface for motion tracking of a piezo-driven micromanipulator. IEEE Trans. Control Syst. Technol. 2010, 18, 798-810. [CrossRef]

17. Liaw, H.C.; Shirinzadeh, B. Constrained motion tracking control of piezo-actuated flexure-based four-bar mech- anisms for micro/nano manipulation. IEEE Trans. Autom. Sci. Eng. 2010, 7, 699-705. [CrossRef]

18. Kanekawa, K.; Matsuya, I.; Sato, M.; Tomishi, R.; Takahashi, M.; Miura, S.; Suzuki, Y.; Hatada, T.; Katamura, R.; Nitta, Y.; et al. An experimental study on relative displacement sensing using phototransistor array for building structures. IEEJ Trans. Electr. Electron. Eng. 2010, 5, 251-255. [CrossRef]

19. Yu, H.; He, X.; Ding, W.; Hu, Y.; Yang, D.; Lu, S.; Wu, C.; Zou, H.; Liu, R.; Lu, C.; et al. A Self-Powered Dynamic Displacement Monitoring System Based on Triboelectric Accelerometer. Adv. Energy Mater. 2017, 7, 1700565. [CrossRef]

20. MacKay, R.E.; Le, H.R.; Clark, S.; Williams, J.A. Polymer micro-grippers with an integrated force sensor for biological manipulation. J. Micromech. Microeng. 2013, 23, 015005. [CrossRef]

21. Kang, D.; Pikhitsa, P.V.; Choi, Y.W.; Lee, C.; Shin, S.S.; Piao, L.; Park, B.; Suh, K.Y.; Kim, T.; Choi, M. Ultrasensitive mechanical crack-based sensor inspired by the spider sensory system. Nature 2014, 516, 222-226. [CrossRef] [PubMed]

22. Wankhar, S.; Kota, A.A.; Selvaraj, D. A versatile stretch sensor for measuring physiological movement using a centre loaded, end-supported load cell. J. Med. Eng. Technol. 2017, 41, 406-414. [CrossRef] [PubMed]

23. Wu, W.; Ren, M.; Pi, B.; Cai, W.; Xu, J. Displacement sensor based on plasmonic slot metamaterials. Appl. Phys. Lett. 2016, 108, 073106. [CrossRef]

24. Zhu, C.; Chen, Y.; Du, Y.; Zhuang, Y.; Liu, F.; Gerald, R.E.; Huang, J. A Displacement Sensor with Centimeter Dynamic Range and Submicrometer Resolution Based on an Optical Interferometer. IEEE Sens. J. 2017, 17, 5523-5528. [CrossRef]

25. Dash, J.N.; Jha, R.; Villatoro, J.; Dass, S. Nano-displacement sensor based on photonic crystal fiber modal interferometer. Opt. Lett. 2015, 40, 467-470. [CrossRef]

26. Liu, F.; Fei, Y.; Xia, H.; Chen, L. A new micro/nano displacement measurement method based on a double-fiber Bragg grating (FBG) sensing structure. Meas. Sci. Technol. 2012, 23, 054002. [CrossRef]

27. Qu, H.; Yan, G.F.; Skorobogatiy, M. Interferometric fiber-optic bending/nano-displacement sensor using plastic dual-core fiber. Opt. Lett. 2014, 39, 4835-4838. [CrossRef] [PubMed]

28. Gao, R.; Lu, D.F.; Cheng, J.; Jiang, Y.; Jiang, L.; Qi, Z.M. Optical Displacement Sensor in a Capillary Covered Hollow Core Fiber Based on Anti-Resonant Reflecting Guidance. IEEE J. Sel. Top. Quantum Electron. 2017, 23, 5600106. [CrossRef]

29. Van de Walle, C.G.; Martin, R.M. Theoretical study of band offsets at semiconductor interfaces. Phys. Rev. B 1987, 35, 8154-8165. [CrossRef]

30. Tomozawa, H.; Numata, K.; Hasegawa, H. Interface states at lattice-matched and pseudomorphic heterostructures, Appl. Surf. Sci. 1992, 60-61, 721-728. [CrossRef]

31. Huang, Y.S.; Westenhoff, S.; Avilov, I.; Sreearunothai, P.; Hodgkiss, J.M.; Deleener, C.; Friend, R.H.; Beljonne, D. Electronic structures of interfacial states formed at polymeric semiconductor heterojunctions. Nat. Mater. 2008, 7, 483-489. [CrossRef] [PubMed]

32. Song, B.S.; Noda, S.; Asano, T. Photonic devices based on in-plane hetero photonic crystals. Science 2003, 300, 1537. [CrossRef] 
33. Rechtsman, M.; Zeuner, J.; Plotnik, Y.; Lumer, Y.; Podolsky, D.; Dreisow, F.; Nolte, S.; Segev, M.; Szameit, A. Photonic Floquet topological insulators. Nature 2013, 496, 196-200. [CrossRef] [PubMed]

34. Fan, Y.-X.; Sang, T.-Q.; Liu, T.; Xu, L.-L.; Tao, Z.-Y. Single-mode interface states in heterostructure waveguides with Bragg and non-Bragg gaps. Sci. Rep. 2017, 7, 44381. [CrossRef] [PubMed]

35. Kim, I.; Iwamoto, S.; Arakawa, Y. Topologically protected elastic waves in one-dimensional phononic crystals of continuous media. Appl. Phys. Exp. 2018, 11, 017201. [CrossRef]

36. Tao, Z.-Y.; Liu, T.; Zhang, C.; Fan, Y.-X. Acoustic extraordinary transmission manipulation based on proximity effects of heterojunctions. Sci. Rep. 2019, 9, 1080. [CrossRef]

37. Gric, T.; Cada, M. Analytic solution to field distribution in one-dimensional inhomogeneous media. Opt. Commun. 2014, 322, 183-187. [CrossRef]

38. Gric, T.; Gorodetsky, A.; Trofimov, A.; Rafailov, E. Tunable plasmonic properties and absorption enhancement in terahertz photoconductive antenna based on optimized plasmonic nanostructures. J. Infrared Millim. Terahz. Waves 2018, 39, 1028-1038. [CrossRef]

39. Zhang, L.; Fan, Y.-X.; Liu, H.; Xu, L.-L.; Xue, J.-L.; Tao, Z.-Y. Hypersensitive and Tunable Terahertz Wave Switch Based on Non-Bragg Structures Filled with Liquid Crystals. J. Light. Technol. 2017, 35, 3092-3098. [CrossRef]

40. Xiao, M.; Ma, G.; Yang, Z.; Sheng, P.; Zhang, Z.Q.; Chan, C.T. Geometric phase and band inversion in periodic acoustic systems. Nat. Phys. 2015, 11, 240-244. [CrossRef]

41. Ellrich, F.; Bauer, M.; Schreiner, N.; Keil, A.; Pfeiffer, T.; Klier, J.; Weber, S.; Jonuscheit, J.; Friederich, F.; Molter, D. Terahertz Quality Inspection for Automotive and Aviation Industries. J. Infrared Millim. Terahz. Waves 2020, 41, 470-489. [CrossRef]

42. Hassan, N.; Chou, C.T.; Hassan, M. eNEUTRAL IoNT: Energy-neutral event monitoring for internet of nano things. IEEE Internet Things J. 2019, 6, 2379-2389. [CrossRef]

(C) 2020 by the authors. Licensee MDPI, Basel, Switzerland. This article is an open access article distributed under the terms and conditions of the Creative Commons Attribution (CC BY) license (http://creativecommons.org/licenses/by/4.0/). 